Mots. Les langages du politique

\title{
À propos du bien et du mal ou Machiavel versus
} Bush

Jean-Claude Zancarini

\section{OpenEdition}

1 Journals

Édition électronique

URL : https://journals.openedition.org/mots/16952

DOI : $10.4000 /$ mots. 16952

ISSN : 1960-6001

Éditeur

ENS Éditions

\section{Édition imprimée}

Date de publication : 1 novembre 2003

Pagination : 169-172

ISBN : 2-84788-043-7

ISSN : 0243-6450

\section{Référence électronique}

Jean-Claude Zancarini, «À propos du bien et du mal ou Machiavel versus Bush », Mots. Les langages du politique [En ligne], 73 | 2003, mis en ligne le 13 octobre 2008, consulté le 23 avril 2022. URL : http:// journals.openedition.org/mots/16952; DOI : https://doi.org/10.4000/mots.16952 


\section{À propos du bien et du mal ou Machiavel versus Bush}

J'ai déjà attiré l'attention, en d'autres lieux, sur certains des aspects de l'usage possible de la boite à outils de Machiavel pour penser le présent. Nous avons, par exemple, Jean-Louis Fournel et moi-même, mis en évidence en quoi la méthode de Machiavel et sa posture éthique (ce que lui même nomme l'offizio di uomo buono, «le devoir d'un homme bon $\left.{ }^{2} »\right)$ pouvaient servir à penser la guerre et son lien avec le politique ou à réfléchir à ce qu'est un acteur politique ${ }^{3}$. Je n'y reviendrai pas ici mais je voudrais insister sur deux points liés à l'actualité et, sans doute, à l'ouverture d'une nouvelle période historique : les attaques suicides du 11 septembre 2001 et la thèse de l'affrontement nécessaire entre un axe du bien et un axe du mal, thèse soutenue, depuis lors, par l'administration Bush. Rappeler à ce propos que pour Machiavel, la religion sert aussi - sinon avant tout - à faire la guerre et que le bien et le mal sont des données relatives, historicisables (et donc que l'on a tout intérêt à historiciser) ne saurait faire du mal ${ }^{4}$ ! Machiavel estime qu'il faut lire sensatamente, c'est-à-dire "de façon sensée », " en donnant du sens »; cet adverbe sensatamente, il l'utilise en effet dans les Discours pour dire comment il faut lire les livres d'histoire (le istorie) et également la Bible. On connait peu le Florentin en exégète biblique et, de fait, il ne cite la Bible qu'une fois, pour rappeler que « celui qui lira la Bible en lui donnant du sens

1. ENS-LSH - 15, Parvis René-Descartes 69366 Lyon cedex 07 -jzancarini@ens-1sh.fr

2. Discours, proemio du livre II.

3. J.-L. Fournel et J.-C. Zancarini, 2000, «L'héritage de Machiavel », Les temps modernes, «La Souveraineté. Horizons et figures de la politique », 610, sept.-nov., Paris ; id., 2000, "Machiavel, Carnéade et les détours de la philosophie », communication présentée au colloque de la SIES «La philosophie italienne»; id., 2002, « Machiavel : la guerre comme horizon de la politique », communication présentée au colloque «Penser la guerre aujourd'hui » (Lyon, mars 2001), Cahiers de la Villa Gillet, no 16, p. 137-151.

4. Les textes de Machiavel utilisés ici sont au premier chef les Discours et Le Prince. Les citations du Prince sont extraites de l'édition que j'ai réalisée avec J.-L. Fournel (2000, Paris, PUF) ; pour les Discours, c'est moi qui traduis. 
(sensatamente), verra que Moïse, pour établir ses lois et ses ordres, a tué un nombre infini d'hommes ». Cette remarque, qui met en évidence le lien originel de la religion avec les armes, le fait que le message divin implique que le sang soit versé, n'est pas marginale dans la pensée de Machiavel. Sa lecture des évènements historiques de son époque, et plus largement de l'histoire de l'Italie, tend précisément à montrer que la responsabilité politique de l'Église romaine est de n'avoir pas appliqué de façon cohérente cette leçon qu'elle aurait pu et dû, d'après lui, tirer de l'expérience de Moïse : l'Église, en dénouant le lien que la religion a avec les armes, a rendu les Italiens sanza religione e cattivi, termes qu'il faut comprendre dans toute leur épaisseur. Sans religion, c'est-à-dire sans ce lien qui permet la constitution d'une communauté apte à combattre et du coup cattivi, «méchants » certes, dans le sens moral du terme, mais aussi "captifs », incapables de faire face à l'ennemi. Cette coupure est en quelque sorte la cause de la faiblesse des armées italiennes : pour Machiavel, l'Église romaine est la principale responsable de l'abandon des «armes propres», ces «populations armées» qu'un lien d'amour unit à leur chef, et qui, seules, permettent les victoires. À l'inverse, on se souviendra du rôle positif de la religion romaine qui, précisément (Discours, I, 11), « servait à commander les armées, à donner du courage à la plèbe, à maintenir les hommes bons, à faire honte aux mauvais (serviva $a$ comandare gli eserciti, a animire la plebe, a mantenere gli uomini buoni, a fare vergognare i rei) ». Dans ce dernier passage, d'ailleurs, Machiavel précise, comme dans celui de la lecture de la Bible, que seuls ceux qui " considèrent bien les histoires romaines (chi considera bene le istorie romane)», se rendent compte de cette importante vérité.

Cette thèse du rôle déterminant de la religion pour faire la guerre incite (devrait inciter) à porter un regard critique sur les discours qui affirment que l'islam ou le christianisme sont, par nature, opposés à la guerre voire à la violence mais aussi sur les discours visant à chercher ailleurs (dans le « nihilisme», pour certains) les raisons des actes de guerre commis au nom d'une religion. Parallèlement à l'énoncé de cette thèse importante, Machiavel déconstruit l'idée de l'antinomie du bien et du mal. Un des passages dans lesquels se fonde cette déconstruction est d'ailleurs un des chapitres des Discours où la réflexion sur la religion catholique tient une place centrale (le chapitre $\mathrm{I}^{\mathrm{er}}$ du livre $\mathrm{III}^{\mathrm{e}}$ ) ; le passage mérite d'être rappelé, dans sa substance : ce chapitre rappelle qu'il faut revenir au début, rénover, revenir aux principes et au principe; Machiavel donne l'exemple de la religion catholique qui, écrit-il, «fut ramenée vers son principe par saint François et saint Dominique », sans quoi, ajoute-t-il, elle aurait disparu (elle aurait été " éteinte ", spenta). Mais le résultat de ce sauvetage par saint François et 
saint Dominique et « leurs ordres nouveaux », gli ordini loro nuovi (terme qui doit faire penser à la fois aux « ordres » franciscains et dominicains, mais aussi aux « ordres » dans le sens de commandements et de modes de fonctionnements transmis à ces derniers par leurs fondateurs) est pour le moins paradoxal : à cause d'eux les "prélats et les chefs de la religion [...] font le pire qu'ils peuvent (fanno il peggio che possono) »... et Machiavel explique que cela advient parce que les saints qui ont sauvé la religion en la renouvelant puis leurs successeurs laissent entendre aux peuples «qu'il est mal de dire du mal du mal (ci dànno loro a intendere come egli è male dir male del male) ». Ce pourrait être un simple bisticcio, un jeu avec les mots, un clin d'œil ironique... Mais il y a, dans le chapitre VIII, 23-25 du Prince, un autre jeu de mots sur le bien et le mal:

Je crois que ceci provient des cruautés mal employées ou bien employées. Bien employées peuvent être appelées - si du mal il est loisible de dire du bien (se del male è licito dire bene) - celles qui se font tout d'un coup, par nécessité de se mettre en sécurité ; et si ensuite on n'y insiste pas mais qu'on les transforme, autant que faire se peut, pour la plus grande utilité des sujets. Mal employées sont celles qui, encore qu'au début il y en ait peu, croissent avec le temps plutôt qu'elles ne s'éteignent.

Mettons en parallèle ces deux formulations (« il est mal de dire du mal du mal », d'une part, « si du mal il est loisible de dire du bien », de l'autre). Les conséquences de ces jeux avec les mots sont de taille : en fait, il faut penser qu'il est loisible de « dire du mal» du mal, mais aussi qu'il est loisible de « dire du mal » du bien... car, précisément, ce qui compte n'est pas de penser en termes de bien ou de mal mais en termes d'effets des choix politiques que l'on effectue... Il est licite de « dire du mal» du mal pour éviter que certains ne fassent « le pire » mais il est tout aussi licite de « dire du mal» du bien quand ce «bien» apparent a aussi pour résultat «le pire». Il ne s'agit pas d'opposer le bien au mal mais d'historiciser - et de politiser - l'un et l'autre. Le syntagme « dire du mal » est alors une métaphore de la pensée critique ; on comprend dès lors pourquoi, dans le prologue de la Mandragola, Machiavel affirmait que «dire du mal » était « son premier métier»:

Io lo ammunisco e dico a questo tale /che sa dir male anch'egli /e come questa fu la sua prim'arte (Je le préviens et je lui dis, à celui-là, qu'il [l'auteur de la pièce] sait lui aussi dire du mal et que ce fut son premier métier).

L'historicité radicale du bien et du mal implique que personne ne puisse à bon droit définir a priori qu'il y a un camp du bien et un camp du mal en évacuant l'analyse politique et historique ; cette historicité implique la responsabilité totale des acteurs du politique qui ne peuvent être justifiés sans 
expliciter les enjeux et les effets attendus de l'agir politique. Cette position machiavélienne n'est en aucune façon rassurante (car elle ne donne aucune réponse a priori), mais elle a un incontestable effet de dévoilement : quand on entend dire qu'une lutte ou une guerre se déroule entre le bien et le mal, ce dévoilement fait apparaitre les questions fondamentales des enjeux et des résultats recherchés de ces actes. La leçon machiavélienne est tout entière dans ce dévoilement : elle donne à la politique, à la réalité parfois violente des rapports de forces et des intérêts, un rôle central ; elle ne permet pas qu'on la camoufle sous l'apparente « douceur des $\operatorname{mots}^{5}$ ».

5. Cette formule est tirée du Dialogo del reggimento di Firenze, écrit en 1521-1525 par Francesco Guicciardini, historien et homme d'État contemporain, et ami de Machiavel. J'ai réalisé, en collaboration avec J.-L. Fournel, des éditions françaises de Francesco Guicciardini : 1988, Avertissements politiques (Ricordi), Paris, Cerf ; 1996, Histoire d'Italie, Paris, Laffont; 1997, Écrits politiques (Discorso di Logrogno et Dialogo del Reggimento di Firenze), Paris, PUF. 